\title{
Statistical Properties of SEE Rate Calculation in the Limits of Large and Small Event Counts
}

\author{
Ray Ladbury \\ Radiation Effects and Analysis Group \\ NASA Goddard Space Flight Center
}

\section{Motivation for a Statistical Approach}

- Goal of SEE rate calculation is to bound the SEE rate

- by how much?

- Estimated rate depends on the fit to the $\sigma$ vs. LET curve

- Should the fit be a "Best" fit or a conservative worst-case fit

- Does the answer depend on error bars

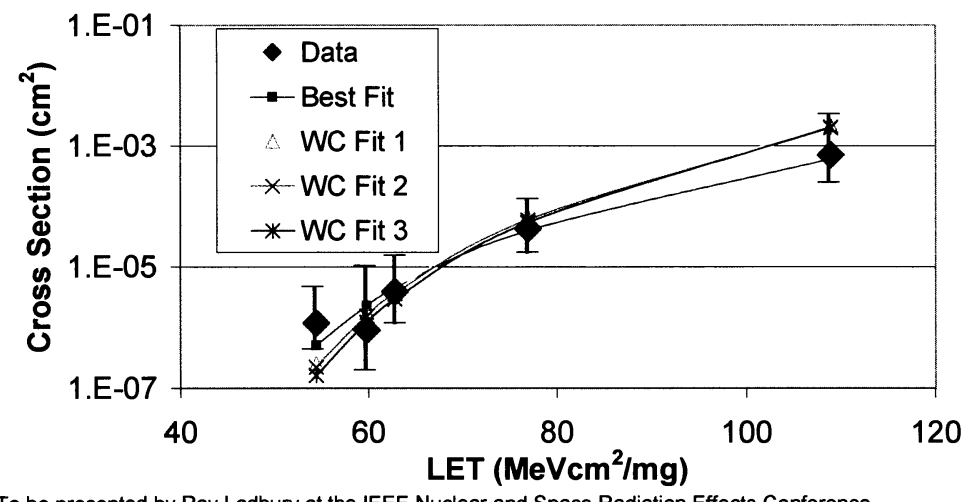

To be presented by Ray Ladbury at the IEEE Nuclear and Space Radiation Effects Conference (NSREC), July 23-27, 2007 and to be published on http://rahome.gsfc.nasa.gov 


\section{Outline}

- Understanding errors on SEE cross sections

- Methodology: Maximum Likelihood and confidence Contours

- Tests with Simulated data

- Applications

\section{Cross sections and Errors}

$$
\sigma\left(\mathrm{LET}_{\mathrm{i}}\right)=\sigma_{\text {lim }} \times\left(1-\exp \left(-\left(\left(\mathrm{LET}_{\mathrm{i}}-\mathrm{LET}_{0}\right) / \mathrm{w}\right)^{\mathrm{s}}\right)\right.
$$

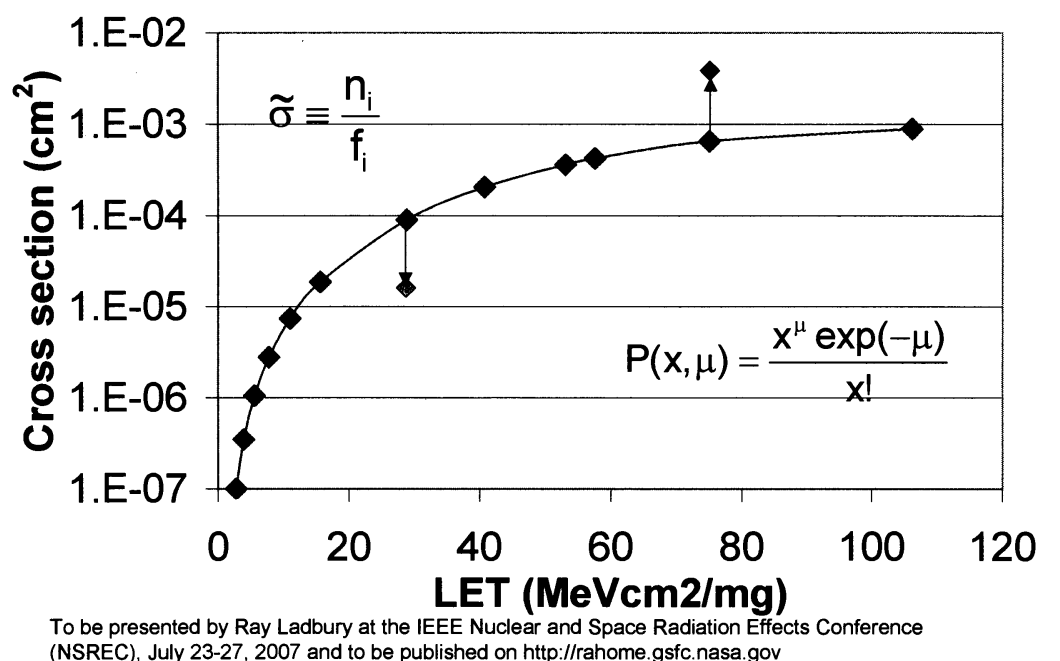




\section{Maximum Likelihood and Confidence}

- Likelihood $\mathcal{L}$ is the product of the probabilities of all our data

- Probabilities of our observations are driven by Poisson fluctuations, so

$\mathcal{L}=\prod_{\mathrm{i}=1}^{\mathrm{n}} \mathrm{P}\left(\mathrm{x}_{\mathrm{i}}, \mu\right)=\mathrm{F}_{\mathrm{i}} \times \sigma_{\text {lim }} \times\left[1-\exp \left\{-\left(\frac{\mathrm{LET}-\mathrm{LET}_{0}}{\mathrm{~W}}\right)^{\mathrm{s}}\right\}\right]$

- Values of ( $\left(\mathrm{LT}_{0}, \sigma_{\text {lim }}, \mathrm{w}\right.$ and $\left.\mathrm{s}\right)$ that maximize $L$ give a best fit to data

- Must be done numerically

- Advantage of Maximum Likelihood is that the ratio of the likelihood for different parameter values gives a measure of their relative probability

$$
\log \left(\mathcal{L}\left(\left\{\mathrm{LET}_{0}^{*}, \sigma_{\text {lim }}^{*}, \mathrm{w}^{*}, \mathrm{~s}^{*}\right\}\right) / \mathcal{L}\left(\left\{\mathrm{LET}_{0}, \sigma_{\text {lim }}, \mathrm{w}, \mathrm{s}\right\}_{\mathrm{bf}}\right)\right) \geq-0.5 \chi^{2}(\mathrm{P}, \mathrm{k})
$$

- This allows construction of confidence contours around our best-fit values

- The highest SEE rate for parameters within a confidence contour will be the WC rate consistent with our data at that confidence level.

- Use Figure of merit (FOM) to select parameter sets $F O M=C \times \sigma_{\text {lim }} / \mathrm{LET}_{0.25}^{2}$

\section{Example with Simulated Data}

Sample Data: Generated with $\mathrm{LET}_{0}=26, \sigma_{\text {lim }}=3.13 \times 10^{-4}, \mathrm{w}=70$ and $\mathrm{s}=2.5$

\begin{tabular}{|l|r|r|r|r|r|r|r|r|r|}
\hline LET $_{\text {EFF }}$ & 7.8 & 11.03 & 15.6 & 28.8 & 40.73 & 53.1 & 57.6 & 75.1 & 106.2 \\
\hline $\begin{array}{l}\text { Cross } \\
\text { Section }\end{array}$ & $0.0 \mathrm{E}+00$ & $0.0 \mathrm{E}+00$ & $0.0 \mathrm{E}+00$ & $1.0 \mathrm{E}-07$ & $6.3 \mathrm{E}-06$ & $2.8 \mathrm{E}-05$ & $4.0 \mathrm{E}-05$ & $1.1 \mathrm{E}-04$ & $2.4 \mathrm{E}-04$ \\
\hline $\begin{array}{l}\text { Events } \\
\text { Observed }\end{array}$ & 0 & 0 & 0 & 1 & 50 & 100 & 100 & 100 & 100 \\
\hline $\begin{array}{l}\text { Effective } \\
\text { Fluence }\end{array}$ & $1.0 \mathrm{E}+07$ & $1.0 \mathrm{E}+07$ & $1.0 \mathrm{E}+07$ & $1.0 \mathrm{E}+07$ & $8.0 \mathrm{E}+06$ & $3.6 \mathrm{E}+06$ & $2.5 \mathrm{E}+06$ & $9.5 \mathrm{E}+05$ & $4.2 \mathrm{E}+05$ \\
\hline
\end{tabular}

\begin{tabular}{|r|r|r|r|r|r|r|r|r|r|}
\hline$\sigma_{\text {lim }}$ LET $_{0}$ & 20 & 21 & 22 & 23 & 24 & 25 & 26 & 27 & 28 \\
\hline $2.13 \mathrm{E}-04$ & -20.41 & -15.29 & -12.74 & -12.82 & -15.57 & -21.10 & -29.51 & -41.02 & -56.28 \\
\hline $2.63 \mathrm{E}-04$ & -33.21 & -21.23 & -12.18 & & & & & -13.17 & -23.93 \\
\hline $3.13 \mathrm{E}-04$ & -62.62 & -43.77 & -28.22 & -16.01 & -7.19 & & & & -8.19 \\
\hline $3.63 \mathrm{E}-04$ & -103.68 & -77.97 & -55.92 & -37.57 & -22.96 & -12.15 & & & \\
\hline $4.13 \mathrm{E}-04$ & -153.39 & -120.81 & -92.26 & -67.77 & -47.37 & -31.11 & -19.05 & -11.37 & -8.65 \\
\hline $4.63 \mathrm{E}-04$ & -209.75 & -170.30 & -135.26 & -104.63 & -78.44 & -56.73 & -39.56 & -27.08 & -19.86 \\
\hline
\end{tabular}




\section{Example with Simulated Data (Cont'd)}

Slice in w-s for best fit cross section and onset LET

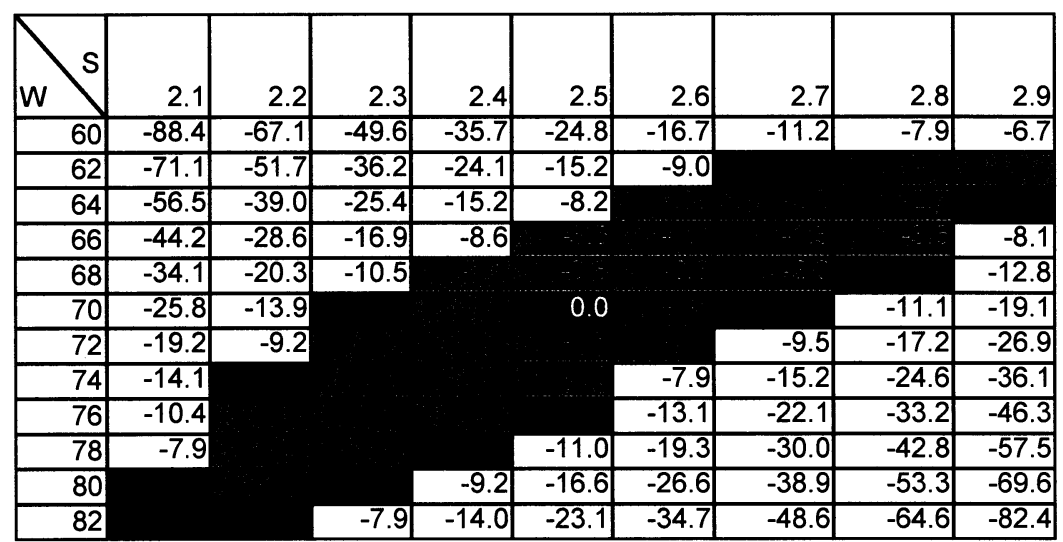

Example with Simulated Data (Cont'd)

\begin{tabular}{|l|r|r|r|r|r|r|r|r|r|}
\hline $\mathrm{LET}_{\mathrm{EFF}}$ & 7.8 & 11.03 & 15.6 & 28.8 & 40.73 & 53.1 & 57.6 & 75.1 & 106.2 \\
\hline $\begin{array}{l}\text { Cross } \\
\text { Section }\end{array}$ & $0.0 \mathrm{E}+00$ & $0.0 \mathrm{E}+00$ & $0.0 \mathrm{E}+00$ & $1.0 \mathrm{E}-07$ & $6.3 \mathrm{E}-06$ & $2.8 \mathrm{E}-05$ & $4.0 \mathrm{E}-05$ & $1.1 \mathrm{E}-04$ & $2.4 \mathrm{E}-04$ \\
\hline $\begin{array}{l}\text { Events } \\
\text { Observed }\end{array}$ & 0 & 0 & 0 & 1 & 2 & 4 & 4 & 4 & 4 \\
\hline $\begin{array}{l}\text { Effective } \\
\text { Fluence }\end{array}$ & $1.0 \mathrm{E}+07$ & $1.0 \mathrm{E}+07$ & $1.0 \mathrm{E}+07$ & $1.0 \mathrm{E}+07$ & $8.0 \mathrm{E}+06$ & $3.6 \mathrm{E}+06$ & $2.5 \mathrm{E}+06$ & $9.5 \mathrm{E}+05$ & $4.2 \mathrm{E}+05$ \\
\hline
\end{tabular}

\begin{tabular}{|c|c|c|c|c|c|c|c|c|c|}
\hline$\sigma_{\text {lim }}$ & 20 & 21 & 22 & 23 & 24 & 25 & 26 & 27 & 28 \\
\hline $1.13 \mathrm{E}-04$ & -6.94 & & & & & & -7.22 & -8.89 & -11.71 \\
\hline $2.13 \mathrm{E}-04$ & -8.89 & & & & & & & & \\
\hline $3.13 \mathrm{E}-04$ & -15.57 & -10.74 & -6.85 & & & & & & \\
\hline 4.13E-04 & -24.29 & -17.52 & -11.96 & -7.55 & & & & & \\
\hline $5.13 \mathrm{E}-04$ & -34.16 & -25.45 & -18.22 & -12.40 & -7.92 & & & & \\
\hline $6.13 \mathrm{E}-04$ & -44.76 & -34.12 & -25.22 & -17.98 & -12.32 & -8.16 & & & \\
\hline
\end{tabular}

To be presented by Ray Ladbury at the IEEE Nuclear and Space Radiation Effects Conference (NSREC), July 23-27, 2007 and to be published on http://rahome.gsfc.nasa.gov 


\section{Example with Simulated Data (Cont'd)}

\begin{tabular}{|c|c|c|c|c|c|c|c|c|c|c|c|c|c|}
\hline$w V$ & 1.7 & 1.9 & 2.1 & 2.3 & 2.5 & 2.7 & 2.9 & 3.1 & 3.3 & 3.5 & 3.7 & 3.9 & 4.1 \\
\hline 45 & -43.2 & -28.5 & -19.4 & -13.6 & -9.9 & -7.6 & & & & & & & \\
\hline 50 & -34.0 & -21.1 & -13.2 & -8.4 & & & & & & & & & \\
\hline 55 & -27.0 & -15.6 & -8.9 & & & & & & & & & & \\
\hline 60 & -21.6 & -11.5 & & & & & & & & & & & -7.4 \\
\hline 65 & -17.4 & -8.5 & & & & & & & & & & -7.9 & -9.8 \\
\hline 70 & -14.1 & & & & 0.0 & & & & & & -8.2 & -10.3 & -12.6 \\
\hline 75 & -11.5 & & & & & & & & & -8.2 & -10.5 & -13.0 & -15.6 \\
\hline 80 & -9.5 & & & & & & & & -8.0 & -10.5 & -13.1 & -15.9 & -18.7 \\
\hline 85 & -8.0 & & & & & & & -7.4 & -10.1 & -12.8 & -15.8 & -18.8 & -21.9 \\
\hline 90 & -6.8 & & & & & & -6.7 & -9.4 & -12.2 & -15.3 & -18.5 & -21.8 & -25.1 \\
\hline 95 & & & & & & & -8.4 & -11.3 & -14.5 & -17.8 & -21.2 & -24.8 & -28.4 \\
\hline 100 & & & & & & -7.2 & -10.1 & -13.3 & \begin{tabular}{l|l|}
-16.7 \\
\end{tabular} & -20.3 & -24.0 & \begin{tabular}{l|l}
-27.7 \\
\end{tabular} & -31.6 \\
\hline 105 & & & & & & -8.7 & -11.9 & -15.4 & -19.0 & -22.8 & -26.7 & -30.7 & -34.7 \\
\hline 110 & & & & & -7.1 & -10.2 & -13.7 & -17.4 & -21.3 & -25.3 & -29.4 & -33.6 & -37.8 \\
\hline 115 & & & & & -8.4 & -11.7 & -15.5 & -19.4 & -23.5 & -27.7 & -32.0 & -36.4 & -40.9 \\
\hline 120 & & & & & -9.7 & -13.3 & -17.2 & -21.4 & -25.7 & -30.1 & -34.6 & -39.2 & -43.8 \\
\hline 125 & & & & -7.6 & -11.0 & -14.8 & -19.0 & -23.4 & -27.9 & -32.5 & -37.2 & -41.9 & -46.7 \\
\hline 130 & & & & -8.6 & -12.3 & -16.4 & -20.7 & -25.3 & -30.0 & -34.8 & -39.7 & -44.6 & -49.6 \\
\hline 135 & & & & -9.7 & -13.6 & -17.9 & -22.4 & -27.2 & -32.1 & -37.0 & -42.1 & -47.2 & -52.3 \\
\hline 140 & & & -7.3 & -10.8 & -14.9 & -19.4 & -24.1 & -29.0 & -34.1 & -39.2 & -44.4 & -49.7 & -55.0 \\
\hline
\end{tabular}

To be presented by Ray Ladbury at the IEEE Nuclear and Space Radiation Effects Conference (NSREC), July 23-27, 2007 and to be published on http://rahome.gsfc.nasa.gov

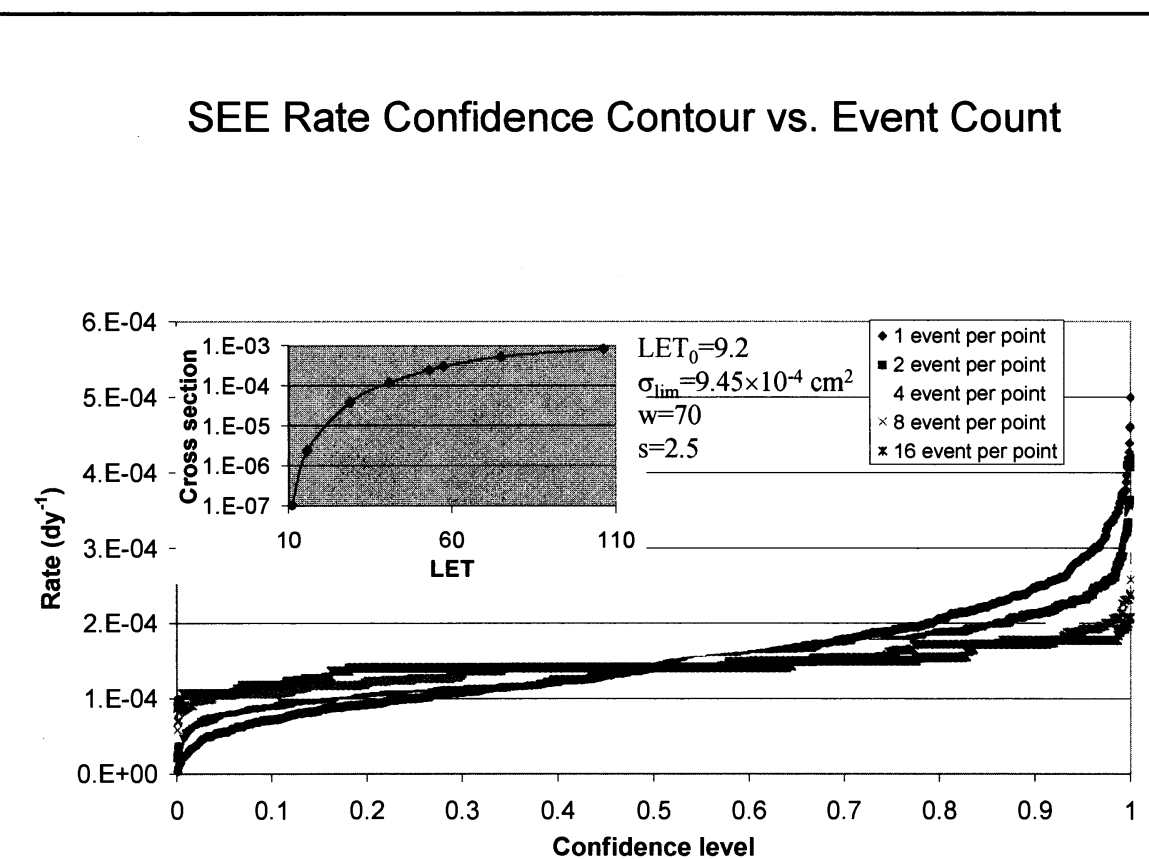

To be presented by Ray Ladbury at the IEEE Nuclear and Space Radiation Effects Conference (NSREC), July 23-27, 2007 and to be published on http://rahome.gsfc.nasa.gov 


\section{How Event Count affects Estimation}

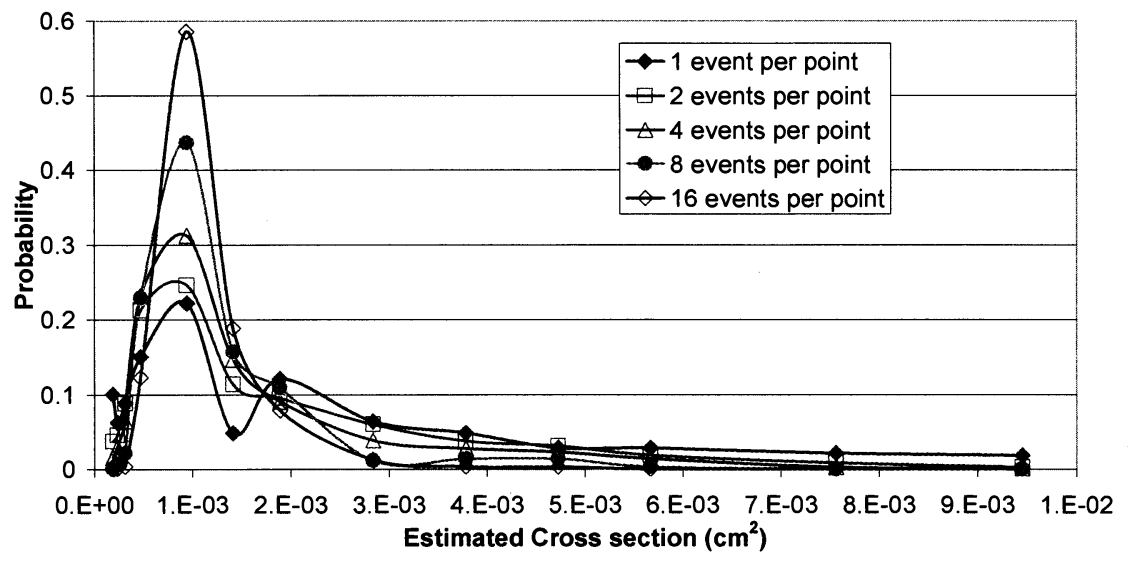

To be presented by Ray Ladbury at the IEEE Nuclear and Space Radiation Effects Conference (NSREC), July 23-27, 2007 and to be published on http://rahome.gsfc.nasa.gov

\section{Applications}

- Determination of part-to-part and lot-to-lot variability

- Poisson errors sometimes give an impression of variability

- Variability is established at significance $1-\alpha$ if the contours for $C L=\alpha$ do not overlap

- Test planning - although we don't know a priori how a test will perform, we can simulate over a range of possible distributions to determine the worst-case dependence of rate error on event count

- Can be used to ensure efficient allocation of test time and resources

- What ions to use?

- How much time for each ion?

- For destructive errors, how many parts to sacrifice at each ion?

- Estimate derating factors as a function of \# of events and confidence level.

- Dividing the data set in post-processing

- Often events of interest to the application emerge only in post-processing

- Example: Some SEFI may pose system-level problems, while others do not

- Example: Only transients larger than a minimum amplitude and longer than a certain duration may pose a threat 


\section{Example: Ultra-long Transients for OP293}

- Testing on Analog Devices OP293 revealed transients up to $1 \mathrm{~ms}$ long

- Boards were already populated and there was no easy replacement

- Circuit analysis revealed concern only for transients $>120 \mu \mathrm{s}$ and $>2 \mathrm{~V}$

\begin{tabular}{|c|c|c|c|}
\hline $\begin{array}{c}\mathrm{LET}_{\text {EFF }} \\
\left(\mathrm{MeVcm}^{2} / \mathrm{mg}\right)\end{array}$ & $\begin{array}{l}\text { Total } \\
\text { transients }\end{array}$ & $\begin{array}{l}\text { Transients }(>120 \\
\mathrm{ms},>2 \mathrm{~V})\end{array}$ & $\begin{array}{l}\text { Cross Section } \\
\left(\mathrm{cm}^{2}\right)\end{array}$ \\
\hline 19.5 & 751 & 1 & $1 \times 10^{-7}$ \\
\hline 28 & 525 & 3 & $3 \times 10^{-7}$ \\
\hline 43 & 679 & 5 & $5 \times 10^{-7}$ \\
\hline 49.5 & 1303 & 0 & 0 \\
\hline 87.5 & 477 & 2 & $2 \times 10^{-7}$ \\
\hline
\end{tabular}

- Testing on Analog Devices OP293 revealed transients up to $1 \mathrm{~ms}$ long

- Boards were already populated and there was no easy replacement

- Circuit analysis revealed concern only for transients $>120 \mu$ s and $>2 V$

\section{Possible Extensions of the Method}

- This work dealt with Poisson errors, where mean $\sigma$ follows a Weibull

- Method can also be extended to model other error including systematic errors

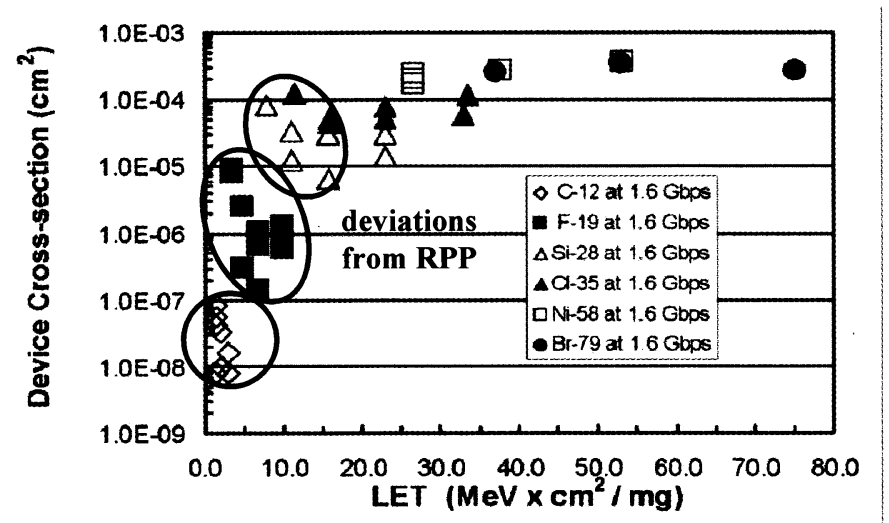

- If we can model it, we can apply this method

- Errors on fluence, deviations from RPP, Weibull form for $\sigma$, and so on.

To be presented by Ray Ladbury at the IEEE Nuclear and Space Radiation Effects Conference (NSREC), July 23-27, 2007 and to be published on http://rahome.gsfc.nasa.gov 


\section{Conclusions}

- Maximum Likelihood methods allow us to bound the SEE rate for a given confidence level IF we can model the errors on $\sigma$ vs. LET curve

- If event counts are high systematic errors will dominate

- Otherwise errors are Poisson

- Allows unambiguous determination of a bounding rate for a given confidence level and data set.

- Applications include

- Determination of part-to-part and lot-to-lot variability

- Test Planning

- Determination of bounding rates for marginal data (e.g. divided data sets)

- Technique can be generalized to include any error we can model

- Other random errors (including part-to-part variations)

- Systematic errors if we can characterize and model them 